OPEN ACCESS

Edited by:

Miguel Viveiros,

New University of Lisbon, Portugal

Reviewed by:

Sukhendu Mandal,

University of Calcutta, India

Marco Pieroni.

University of Parma, Italy

${ }^{*}$ Correspondence:

Chunling Xiao

xiaoc1304@163.com

${ }^{\dagger}$ These authors have contributed equally to this work

Specialty section:

This article was submitted to Antimicrobials, Resistance

and Chemotherapy,

a section of the journal

Frontiers in Microbiology

Received: 14 June 2019 Accepted: 16 December 2019

Published: 15 January 2020

Citation:

Meng J, Gao P, Wang X, Guan Y, Liu Y and Xiao C (2020) Digging

Deeper to Save the Old

Anti-tuberculosis Target: D-Alanine-D-Alanine Ligase With

a Novel Inhibitor, IMB-0283.

Front. Microbiol. 10:3017.

doi: 10.3389/fmicb.2019.03017

\section{Digging Deeper to Save the Old Anti-tuberculosis Target: D-Alanine-D-Alanine Ligase With a Novel Inhibitor, IMB-0283}

\author{
Jianzhou Meng ${ }^{1 t}$, Peng Gao ${ }^{2 t}$, Xiao Wang ${ }^{1}$, Yan Guan', Yishuang Liu ${ }^{1}$ and \\ Chunling Xiao ${ }^{1 *}$
}

${ }^{1}$ Institute of Medicinal Biotechnology, Chinese Academy of Medical Sciences and Peking Union Medical College, Beijing, China, ${ }^{2}$ Department of Microbiology, Li Ka Shing Faculty of Medicine, The University of Hong Kong, Hong Kong, China

The emergence of drug-resistant Mycobacterium tuberculosis (Mtb) has hampered treatments for tuberculosis, which consequently now require novel agents to overcome such drug resistance. The genetically stable D-alanine-D-alanine ligase A (DdIA) has been deemed as an excellent therapeutic target for tuberculosis. In the present study, a competitive inhibitor (IMB-0283) of DdlA was obtained via high-throughput screening. The minimum inhibitory concentrations (MIC) of IMB-0283 for the standard and clinical drug-resistant Mtb strains ranged from 0.25 to $4.00 \mu \mathrm{g} / \mathrm{mL}$, whereas the conventional inhibitor of DdlA, D-cycloserine (DCS), only inhibited the growth of the standard Mtb strain at $16 \mu \mathrm{g} / \mathrm{mL}$. The lethal effect of IMB-0283 on Mtb was found to act intracellularly in a DdIA-dependent manner. Specifically, IMB-0283 prevented the synthesis of neonatal cell walls but did not damage mature cell walls. Compared with those of DCS, IMB0283 exhibited lower cytotoxicity and a higher selective index (SI). At the same dosages of treatment, IMB-0283 reduced bacterial load (log CFU/mL) in an acute animal model from 5.58 to 4.40 , while DCS did not yield any such treatment efficacy. Taken together, the lower cytotoxicity and more efficacious in vivo activity of IMB-0283 suggest that it is a promising lead compound for antituberculosis drug development.

Keywords: Mycobacterium tuberculosis, drug-resistance, D-alanine-D-alanine ligase, inhibitor, D-cycloserine

\section{INTRODUCTION}

Tuberculosis (TB) is remains as a high-burden disease and has claimed millions of lives all over the world due to infections of Mtb. Multidrug-resistant TB occurs in 3.5\% of new cases and $18 \%$ of previously treated cases, and extensive drug-resistant $\mathrm{TB}$ has been on the rise in recent years (WHO, 2018). Hence, conventional chemotherapeutics have become inert in attempts to combat

Abbreviations: DCS, D-cycloserine; DdlA, D-alanine-D-alanine ligase A; INH, isoniazid; MIC, minimum inhibitory concentrations; Mtb, Mycobacterium tuberculosis; RMP, rifampin; SI, selective index; TB, tuberculosis. 
drug-resistant Mtb infections, which consequently now require novel agents to overcome such drug resistance.

Validating whether a therapeutic target with a novel mechanism is worthy of further drug discovery and development is difficult, time-consuming, and requires tremendous resources. To efficiently utilize limited resources, we aimed to leverage a known druggable target since several old drug targets have been previously recognized as promising candidates. Our selection criteria for such old druggable targets were as follows: (1) genetic stability of the target, which may lessen the probability of drug resistance; and (2) the present inhibitor of the target is restricted due to severe side effects but not drug resistance. D-alanineD-alanine ligase A (DdlA, EC 6.3.2.4, and Rv2981c), the target of DCS (Halouska et al., 2014), is an excellent drug target for treating TB since its mutation rate to generate resistant strains is much smaller than that of other targets (McGrath et al., 2014). However, the neurological and psychiatric side effects of DCS limit its clinical application (Kass and Shandera, 2010). Therefore, it is necessary to exploit novel anti-TB compounds that target Mtb DdlA. In the present study, a safe and low-toxicity inhibitor of DdlA was obtained with potent anti-TB activity both in vitro and in vivo.

\section{MATERIALS AND METHODS}

\section{Bacteria and Plasmids}

All chemicals used in this study were purchased from Sigma (Sigma-Aldrich, St. Louis, MO, United States) unless otherwise stated. Mtb H37Rv (ATCC27294) and other clinical drug-resistant strains conserved by the Chinese Center For Disease Control And Prevention were cultured in Middlebrook 7H9 broth (supplemented with glycerol and polysorbate 80) in combination with Middlebrook albumin-dextrose-catalase (ADC) enrichment or 7H10 agar solid media supplemented with oleic acid-albumin-dextrose-catalase (OADC) enrichment $($ ADC $+0.003 \%$ oleic acid) (Allen, 1998). Escherichia coli (E. coli) DH5 $\alpha$ and E. coli BL21 (DE3) plyS (TransGen Biotech, Inc., Beijing, China) were cultured in Luria-Bertani (LB) broth or on LB agar plates. Plasmid pET28a (+) was conserved by our lab and plasmid pAZI9479 was kindly gifted by Professor Francesca Forti (Forti et al., 2009). Kanamycin was added at concentrations of $100 \mu \mathrm{g} / \mathrm{mL}$ for E. coli, while hygromycin was added at $200 \mu \mathrm{g} / \mathrm{mL}$ for Escherichia coli and at $100 \mu \mathrm{g} / \mathrm{mL}$ for Mtb. Isopropyl $\beta$-D-1thiogalactopyranoside (IPTG) was used as an inducer to express DdlA in E. coli BL21 (DE3) plyS, and pristinamycin (Santa Cruz Biotech, Santa Cruz, CA, United States) was used to induce gene expression in Mtb.

\section{Molecular Manipulations}

The Mtb H37Rv genome was extracted from the log phase cells as previously described in Mtb protocols (Gordhan and Parish, 2001). All PCR reagents were purchased from TransGen Biotech. The primers for amplification $d d l$ A were designed using software primer 5.0 based on the sequence in the NCBI database (GenBank accession number: 888415). The primers $d d l \mathrm{~A}$ F (5'-AAAAGAATTCGTGAGTGCTAACGACCGGC-3') and $d d l$ A R (5'-AAAAAAGCTTCTAGTGCAGGCCCACGCCG$\left.3^{\prime}\right)$ were used to amplify the whole fragment of $d d l \mathrm{~A}$ for protein expression, and primers ddlAM $\mathrm{F}$ (5'AATTCCATGGGTGAGTGCTAACGACCGGC-3' ${ }^{\prime}$ ) and $d d l$ AM R (5'-CGCCCATATGGGGTTTGACGAACACCGGTA-3') were used to synthesize the former fragment of $d d l$ A for constructing a conditional mutant strain. The lineated parts of the sequences denote the limited digestion sites (EcoRI, HindIII, NcoI, and NdeI).

The purified PCR product of the $d d l \mathrm{~A}$ gene was cloned into plasmid pET28a (+)EcoRI-HindIII sites to generate pET28a (+)$d d l \mathrm{~A}$; the fragment of $d d l \mathrm{AM}$ was cloned into pAZI9479 NcoINdeI sites to obtain pAZI9479-DM. Sequencing was subsequently performed to ensure that there were no mutations.

\section{Construction of the Conditional Mutant Bacteria}

The bacteria and plasmids were treated as previously described (Meng et al., 2015). First, $1 \mu \mathrm{g}$ of UV-illuminated plasmid pAZI9479-DM (no more than $5 \mu \mathrm{L}$ ) was mixed with $200 \mu \mathrm{L}$ of competent cells in a $0.2-\mathrm{cm}$ electroporation cuvette (Biorad, Hercules, CA, United States), and the cuvette was pulsed at a strength of $2.5 \mathrm{kV}, 25 \mathrm{uF}$, and $1000 \Omega$ resistance with a Gene Pulser Xcell (Bio-rad). These bacteria were resuscitated in $5 \mathrm{~mL}$ of $7 \mathrm{H} 9$ broth for $24 \mathrm{~h}$ at $37^{\circ} \mathrm{C}$ and were then spread on $7 \mathrm{H} 10$ plates containing $0.5 \mu \mathrm{g} / \mathrm{mL}$ of pristinamycin and $100 \mu \mathrm{g} / \mathrm{mL}$ of hygromycin that were incubated at $37^{\circ} \mathrm{C}$ for 4 weeks until colonies formed. Plasmid-positive bacteria were determined via sequencing, and the resultant mutant strain was designated as Mtb-KD.

\section{Expression and Purification of DdIA}

The log-phase E. coli BL21 (DE3) pLysS-bearing plasmid pET28a $(+)$-ddlA were induced by IPTG (final concentration of $0.3 \mathrm{mM}$ ) at $28^{\circ} \mathrm{C}$ for $8 \mathrm{~h}$, and the recombinant protein carrying a hexahistidine tag at the $\mathrm{N}$-terminal was purified by $\mathrm{Ni}^{2+}$ ionaffinity chromatography using a bouncing gradient of 40-100200-400 mM imidazole in washing buffer $(20 \mathrm{Mm}$ of Tris- $\mathrm{HCl}$, $\mathrm{pH}$ of $8.0,500 \mathrm{mM}$ of $\mathrm{NaCl}, 1 \mathrm{mM}$ of dithiothreitol). The eluted fractions were analyzed by SDS-PAGE and were visualized with Coomassie Brilliant Blue R-250 gel staining. The purified DdlA was desalted on a PD-10 column (GE Healthcare, Piscataway, NJ, United States), concentrated through a $10-\mathrm{kDa}$ cut-off Millipore Centricon device (Millipore, Billerica, MA, United States), and was stored at $-80^{\circ} \mathrm{C}$ with $50 \%$ glycerol. The concentration of the enzyme was identified via the BCA protein assay kit (TransGen Biotech).

\section{Kinetic Analysis of DdIA}

The activity of DdlA was monitored by coupling with pyruvate kinase (PK) and lactate dehydrogenase (LDH; Sigma-Aldrich) to detect the release of ADP. Reactions were proceeded in 96-well plates (at $37^{\circ} \mathrm{C}$ ) containing $50 \mathrm{mM}$ of Tris- $\mathrm{HCl}(\mathrm{pH} 8.0), 10 \mathrm{mM}$ of $\mathrm{MgCl}_{2}, 10 \mathrm{mM}$ of $\mathrm{KCl}, 1 \mathrm{mM}$ of dithiothreitol, $250 \mu \mathrm{M}$ of ATP, $1000 \mu \mathrm{M}$ of D-alanine (D-Ala), $1 \mathrm{mM}$ of phosphoenolpyruvic acid (PEP), $0.5 \mathrm{mM}$ of NADH, $2 \mu \mathrm{g}$ of DdlA, $1 \mathrm{U}$ of pyruvate 
kinase (PK) and $1 \mathrm{U}$ of lactate dehydrogenase (LDH). The assay system was monitored via a Perkin Elmer EnSpire ${ }^{\circledR} 2300$ Multimode Plate Reader (PerkinElmer, MA, United States) by detecting decreases in $\mathrm{NADH}$.

The calibration curve of the ultraviolet absorption of $\mathrm{NADH}$ at $340 \mathrm{~nm}$ to its concentrations was fabricated before studying the enzymatic kinetics of DdlA. The kinetic parameters of DldA were determined using serial twofold dilutions of one substrate (from $1 \mathrm{mM}$ to $31.25 \mu \mathrm{M}$ ), while the other substrate was held constant at $1 \mathrm{mM}$. Km and Vmax values were calculated using non-linear regression of the Michaelis-Menten model in GraphPad Prism 5.0 (GraphPad Software, Inc., San Diego, CA, United States). Kcat was calculated based on the molecular weight of DdlA (42 kDa).

\section{Screening of Inhibitors}

A high-throughput screening assay was designed according to kinetic parameters. Systems containing $1 \mu \mathrm{L}$ of dimethyl sulfoxide (DMSO) were used as negative controls, while the positive control systems contained heat-inactivated DdlA. Specifically, $1 \mu \mathrm{L}$ of samples diluted at $2 \mathrm{mg} / \mathrm{mL}$ were added to a 96-well plate with a final concentration of $20 \mu \mathrm{g} / \mathrm{mL}$. Parameters signal window, $Z^{\prime}$ factor, and an assay variability ratio were used to assess the reliability of the model (Iversen et al., 2006). Via this model, we screened through 150,000 synthetic compounds. The inhibition rate was calculated as follows:

$$
\mathrm{IR}=\left(1-\frac{\mathrm{A} p-\mathrm{A} s}{\mathrm{~A} p-\mathrm{A} n}\right) \times 100 \%
$$

in which the IR, An, As, and Ap denote the inhibition rate and the ultraviolet absorption of the negative control, sample, and positive control, respectively. The IR threshold was defined at $30 \%$. The IRs of inhibitors in twofold serial dilutions to DdlA were detected to calculate their $\mathrm{IC}_{50}$ values using the non-linear regression module of GraphPad Prism 5.0. The reaction rates of systems containing various concentrations of substrate $(100,200$, $300,400,500$, and $600 \mu \mathrm{M})$ and inhibitors $(10$ or $40 \mu \mathrm{g} / \mathrm{mL}$ ) were detected to determine their inhibitory modes. Lineweaver-Burk plots and Dixon plots were applied to analyze the results.

\section{Antibacterial Activity in vitro}

The minimal inhibitory concentration (MIC) of IMB-0283 (J\&K Chemical Company, Beijing, China, synthesized by Enamine) to Mtb was determined as previously described (Darby and Nathan, 2010). Briefly, the mid-log phase H37Rv and the other clinical drug-resistant strains were ultrasonically suspended and adjusted to a final concentration of $1-2 \times 10^{5} \mathrm{CFU} / \mathrm{mL}$ in $7 \mathrm{H} 9$ broth. Then, $100 \mu \mathrm{L}$ of suspension was exposed to the compound in serial twofold dilutions, from $128 \mu \mathrm{g} / \mathrm{mL}$ to $0.063 \mu \mathrm{g} / \mathrm{mL}$, in 96-well plates in triplicate. INH and RMP were used as positive controls. After 2 weeks of incubation at $37^{\circ} \mathrm{C}$, the viability of bacteria was detected using a resazurin microtiter assay (Montoro et al., 2005). The synergistic effects of IMB-0283 with INH and RMP were tested as previously described (Shen et al., 2010). INH and RMP were twofold diluted from $16 \mu \mathrm{g} / \mathrm{mL}$ to $0.008 \mu \mathrm{g} / \mathrm{mL}$, and their MIC values to $\mathrm{H} 37 \mathrm{Rv}$ were determined when the concentration of IMB- 0283 was set at $0.25 \times$ MIC.
The sensitivity of Mtb-KD cultured with higher or lower concentrations of pristinamycin $\left(0.1\right.$ and $\left.0.1 \times 10^{-4} \mu \mathrm{g} / \mathrm{mL}\right)$ to IMB-0283 was tested to confirm if it interacted with DdlA in bacteria, and DCS was used as a positive control.

\section{Morphology of Mtb Treated With IMB-0283}

The log phase of Mtb H37Rv was treated with $0.35 \mu \mathrm{g} / \mathrm{mL}$ of IMB-0283 and was incubated for $12 \mathrm{~h}$ or 7 days, and Mtb morphological transformations were detected as previously described (Shen et al., 2010). The washed bacterial pellets were spotted on Si chips (Ted Pella, Inc., Redding, CA, United States) and were fixed with $2.5 \%$ glutaraldehyde. The chips were washed and dehydrated in a graded ethanol series. Samples were coated with $\mathrm{Au} / \mathrm{Pd}$ (E-1045 ion sputter coater, Hitachi High Technologies Co., Tokyo, Japan) after being critical point-dried (Bal-Tec CPD 030 Critical Point Dryer, Bal-Tec AG, Liechtenstein, Germany). The bacteria morphologies were surveyed by a Quanta200 scanning electron microscope (FEI, Oregon, United States).

\section{Cytotoxicity Assay}

HepG2 cells (ATCCHB-8065) and Vero green-monkey kidney cells (ATCCC1008) were cultured in Dulbecco's modified eagle's medium (DMEM) supplemented with $10 \%$ fetal bovine serum (FBS) and $1 \%$ antibiotics (100 U/mL penicillin), and were incubated at $5 \% \mathrm{CO}_{2}$ in a humidified atmosphere at $37^{\circ} \mathrm{C}$ (Heracell 150, Thermo Electron Corp., Waltham, MA, United States). After $24 \mathrm{~h}$ of incubation, the adhered exponential cells $\left(1 \times 10^{5}\right.$ cells $\left./ 100 \mu \mathrm{L}\right)$ were washed three times with fresh medium. IMB-0283 was added at a triple dilution from $900 \mu \mathrm{g} / \mathrm{mL}$ to $1.23 \mu \mathrm{g} / \mathrm{mL}$ with the medium, while DCS was diluted from $3000 \mu \mathrm{g} / \mathrm{mL}$ to $4.11 \mu \mathrm{g} / \mathrm{mL}$. DMSO (1\%) was added to induce a solvent effect, and normal-growth cells were used as negative controls. 3-[4, 5-dimethylthiazol-2-yl]-2, 5diphenyl tetrazolium bromide (MTT) were used to detect cell viability at 2 days later (Correia et al., 2014). TC 50 values were calculated using non-linear regression (curve fit) of the log inhibitor vs. the normalized response-variable slope module of GraphPad Prism 5.0.

\section{IMB-0283 Activity in a Mouse Model of TB}

Specific pathogen-free (SPF) male Balb/c mice were purchased from the Institute of Laboratory Animal Sciences at the Chinese Academy of Medical Sciences and Peking Union Medical College. All animal experiments were supervised and approved by the Institutional Animal Care and Use Committee of the Institute of Medicinal Biotechnology. Twenty-six mice (6-8 weeks old, $18-20 \mathrm{~g}$ ) were infected with Mtb H37Rv (about $100 \mathrm{CFU}$ ) via the 099C A4224 Inhalation Exposure System (Glas-col, Terre Haute, IN, United States). At 15 days after inoculum, two mice were anesthetized and decapitated to confirm the success of infection. DCS, INH, IMB-0283, and vehicle (0.5\% CMC-Na) were administered through a gavage route (six mice per group). All chemicals were dosed at $25 \mathrm{mg} / \mathrm{kg}$. Mice were sacrificed after 
15 administrations (five times per week) to quantify pulmonary bacterial loads via counting CFUs.

\section{RESULTS}

\section{Expression, Purification, and Kinetic Characterization of DdIA}

The protein, DdlA, was purified from E. coli BL21 (DE3) pLysSharboring pET28a (+)-ddlA via immobilized metal affinity chromatography with $\mathrm{Ni}^{2+}$-NTA agarose. The purified protein was verified by SDS-PAGE (Figure 1), and DdlA (42 kDa) was visualized between 35 and $48 \mathrm{kDa}$.

The initial reaction rate of DdlA was determined by correlating the concentrations of ATP and D-Ala to the UV absorbances of $\mathrm{NADH}$. The reaction rates of DdlA affected by concentrations of substrates were detected to calculate its enzymatic kinetic parameters (Figure 2 and Table 1). The Kms for ATP and D-Ala were $450.6 \mu \mathrm{M}$ and $1780 \mu \mathrm{M}$, respectively, and their Vmax values were $20.29 \mu \mathrm{Mmin}^{-1}$ and $36.61 \mu \mathrm{Mmin}^{-1}$, respectively. Kcat values, calculated by dividing Vmax with the enzyme concentration, for ATP and D-Ala were $405.8 \mathrm{~min}^{-1}$ and $732.19 \mathrm{~min}^{-1}$, respectively. These results were consistent with those reported previously (Prosser and de Carvalho, 2013).

\section{HTS Assay and Identification of DdIA Inhibitor}

The A high-throughput screening assay was established according to the enzymatic parameters of DdlA. The signal window, $Z^{\prime}$-factor and assay variability ratio of the screening model were $12.87,0.76$, and 0.24 , respectively, indicating that it was a reliable screening model. Via this model, 48 compounds (inhibition ratio $\geq 30 \%$ ) were obtained from our library (the positive rate was $0.032 \%)$. Among these inhibitors, IMB-0283

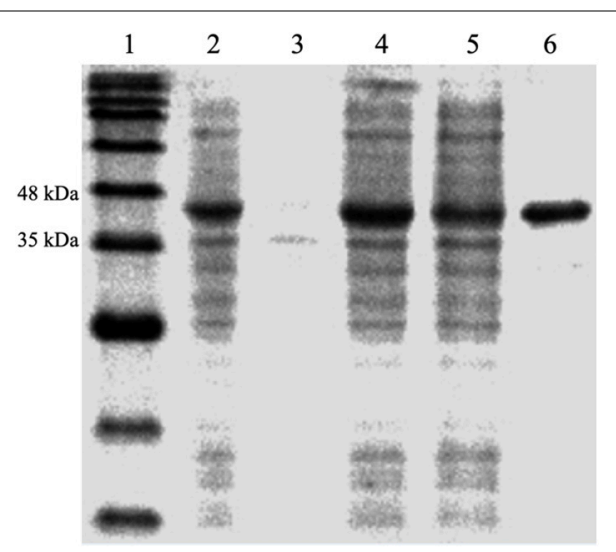

FIGURE 1 | SDS-PAGE analysis of the expression and purification of enzyme DdIA. Lane 1 represents the pre-sharp protein standard. Lane 2 shows the whole protein of the recombinant cell. Lane 3 shows the precipitate protein. Lane 4 shows the supernatant fluid. Lane 5 shows the effluent liquid. Lane 6 shows the purified DdIA.

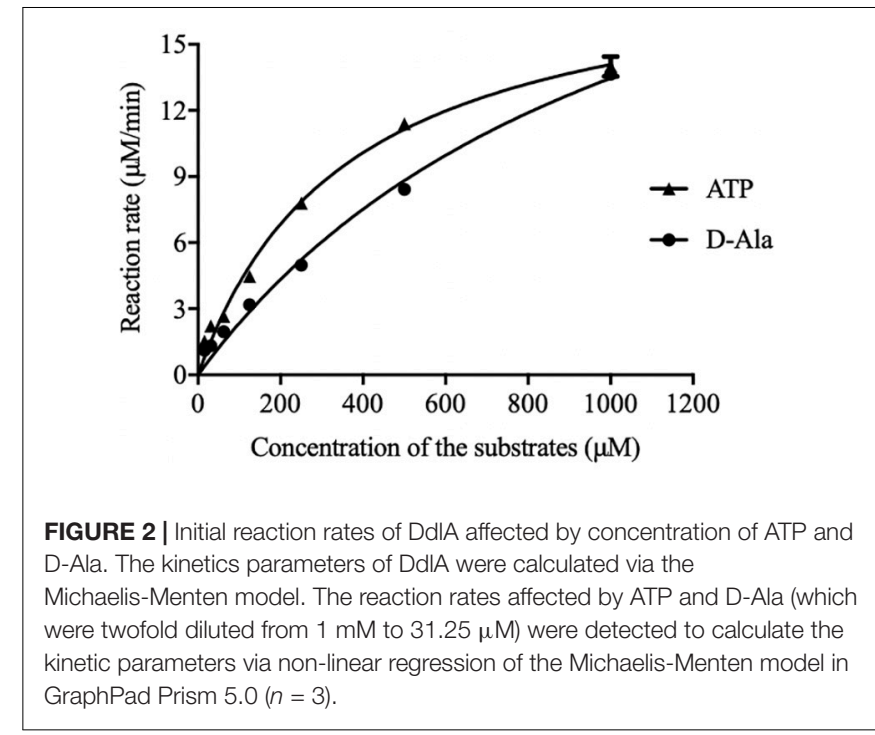

TABLE 1 | Kinetic parameters of DdIA.

\begin{tabular}{lccc}
\hline Substrate & $\mathbf{K m}(\boldsymbol{\mu} \mathbf{M})$ & $\mathbf{V m a x}\left(\boldsymbol{\mu} \mathbf{M m i n}{ }^{-\mathbf{1}}\right)$ & Kcat $^{\mathbf{a}}\left(\mathbf{m i n}^{\mathbf{- 1}}\right)$ \\
\hline ATP & $359.90 \pm 30.53$ & $19.15 \pm 0.705$ & $383.00 \pm 14.14$ \\
D-Ala & $1113.00 \pm 153.90$ & $28.44 \pm 2.444$ & $568.79 \pm 48.93$
\end{tabular}

Kcat ${ }^{a}$ was calculated via Vmax/[E].

displayed the most potent inhibitory activity to DdlA, with an $\mathrm{IC}_{50}$ of $6.16 \mu \mathrm{M}$ (Figure 3).

The reaction rates of the assays affected by various concentrations of inhibitors and substrates were measured to analyze inhibitory modes and to calculate Ki values. IMB0283 competed with both substrates of DdlA with Ki values of $4.444 \mu \mathrm{M}$ (ATP) and $32.647 \mu \mathrm{M}$ (D-Ala) (Figure 4). Ki values of DCS for these substrates were $106.599 \mu \mathrm{M}$ and $182.108 \mu \mathrm{M}$, indicating that IMB-0283 had a better affinity to DdlA compared to that of DCS.

\section{Anti-tuberculosis Activity of IMB-0283}

IMB-0283 potently inhibited H37Rv at an MIC of $0.5 \mu \mathrm{g} / \mathrm{mL}$, while the MIC of DCS was $16 \mu \mathrm{g} / \mathrm{mL}$. IMB-0283 also displayed antibacterial activity to several clinical strains, with MICs ranging from $0.5 \mu \mathrm{g} / \mathrm{mL}$ to $4.0 \mu \mathrm{g} / \mathrm{mL}$ (Table 2), while DCS did not show any inhibitory effect. These results indicated that IMB-0283 had no cross-resistance with that of conventional anti-TB drugs.

The MIC of INH against H37Rv was reduced eightfold, from $0.125 \mu \mathrm{g} / \mathrm{mL}$ to $0.016 \mu \mathrm{g} / \mathrm{mL}$, when combined with IMB- 0283 at $0.125 \mu \mathrm{g} / \mathrm{mL}$. For RMP, the MIC decreased from $0.063 \mu \mathrm{g} / \mathrm{mL}$ to $0.016 \mu \mathrm{g} / \mathrm{mL}$. This finding suggests that there is a synergistic effect between IMB-0283 and either INH or RMP.

\section{IMB-0283 Intracellularly Interacts With DdIA}

A fragment (about $600 \mathrm{bp}$ ) of $d d l$ A starting from ATG was cloned into pAZI9479 to generate pAZI9479KD. The mutant Mtb-KD was generated by inserting pAZI9479KD into the Mtb 

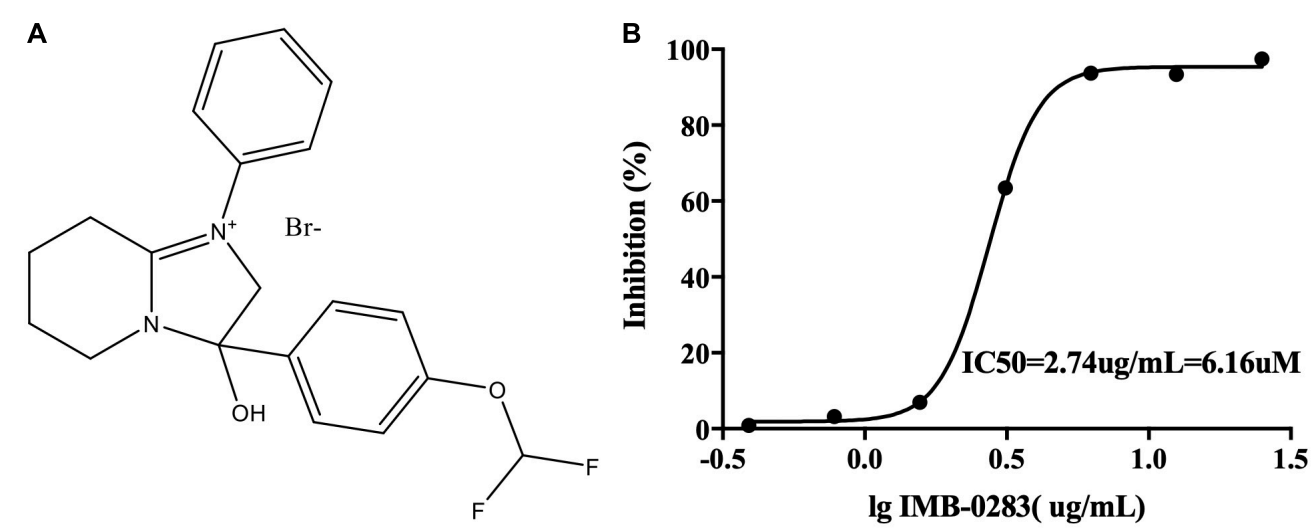

FIGURE 3 | The structure of IMB-0283 and its IC 50 value for DdIA. (A) The structure of IMB-0283. (B) The IC 50 value of IMB-0283 to DdIA. Results are presented as the mean $\pm \operatorname{SD}(n=3)$.

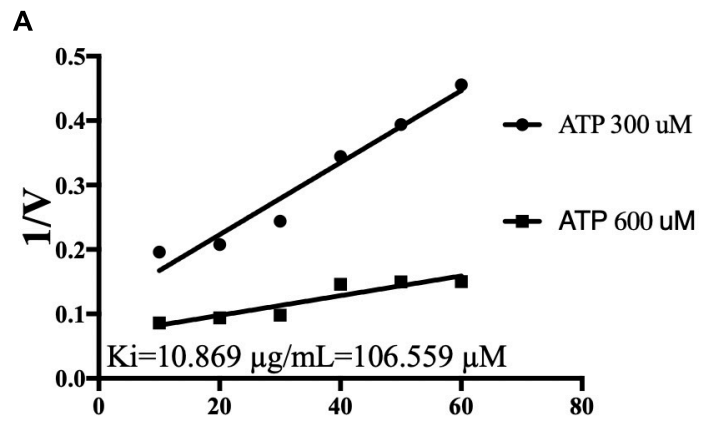

D-cycloserine (ug/mL)

C

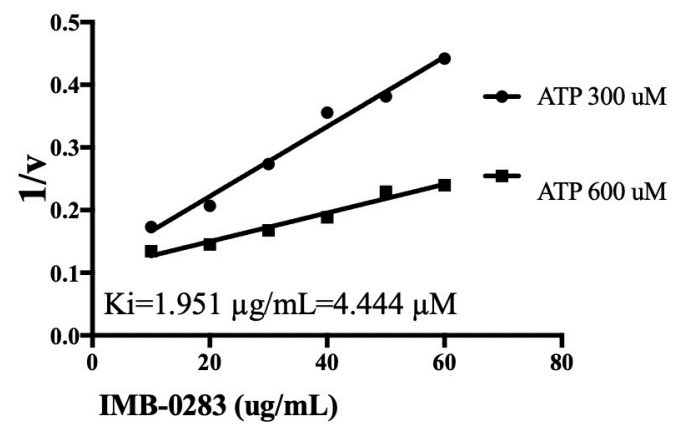

B

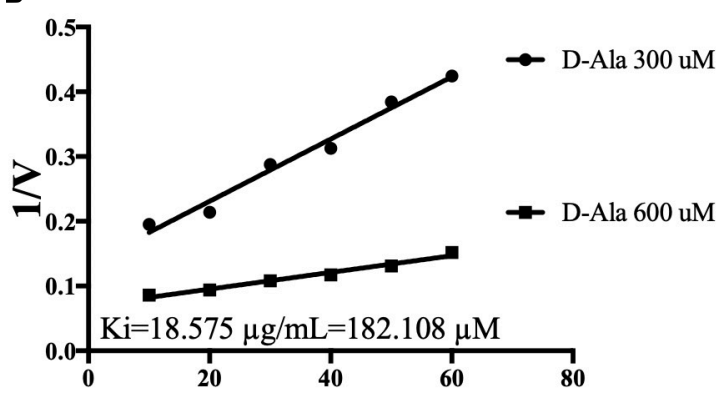

D-cycloserine (ug/mL)

D

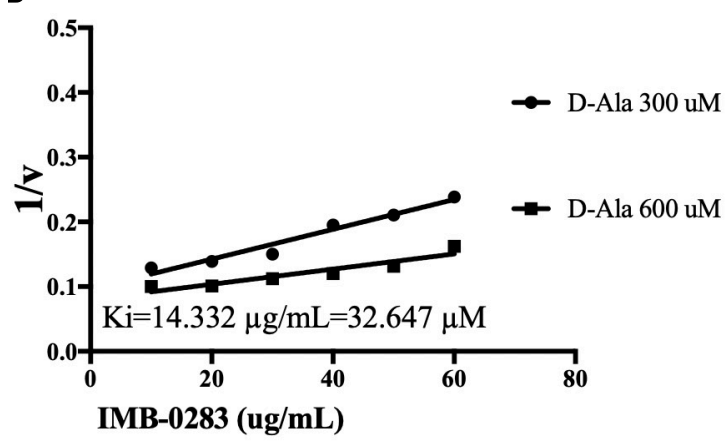

FIGURE 4 | Ki values of inhibitors calculated by Dixon-plots for DdIA. Reactions were monitored when inhibitors varied from 10 to 60 $\mu$ g/mL, while one substrate was fixed at $1000 \mu \mathrm{M}$ and the other substrate was either 300 or $600 \mu \mathrm{M}(n=3)$. Panels $\mathbf{( A , B ) ~ s h o w ~ t h e ~ K i ~ v a l u e s ~ o f ~ D C S ~ t o ~ D d l A . ~ P a n e l s ~ ( C , D ) ~ s h o w ~ t h e ~ K i ~ v a l u e s ~}$ of IMB-0283 to the enzyme.

chromosome. In this mutant strain, the expression of $d d l \mathrm{~A}$ was under the control of pristinamycin. A higher concentration of pristinamycin $(0.1 \mu \mathrm{g} / \mathrm{mL})$ led to overexpression of $d d l A$, while a lower concentration of pristinamycin $\left(0.1 \times 10^{-4} \mu \mathrm{g} / \mathrm{mL}\right)$ corresponded to knock-down of $d d l A$. Figure $5 \mathbf{A}$ shows that the mutant bacteria were not able to grow on the $7 \mathrm{H} 10$ plates without pristinamycin, while the growth of wild-type Mtb was independent of pristinamycin. These results demonstrate that DalA was essential for Mtb. Susceptibilities of Mtb-KD cultured with different concentrations of inducer to IMB0283 were detected to confirm whether the lethal effect was dependent on inhibiting DdlA intracellularly. When the $d d l A$ gene was knocked-down, IMB-0283 and DCS inhibited the mutant at $0.25 \mu \mathrm{g} / \mathrm{mL}$ and $1 \mu \mathrm{g} / \mathrm{mL}$, respectively. Their MIC values climbed to $2 \mu \mathrm{g} / \mathrm{mL}$ and $32 \mu \mathrm{g} / \mathrm{mL}$, respectively, when the $d d l$ a gene was overexpressed (Figure 5B). Consistent 
TABLE 2 | Anti-tuberculous activity of IMB-0283.

\begin{tabular}{lcccccccc}
\hline Mtb strains & \multicolumn{9}{c}{ Drug sensitivity background* } & \multicolumn{1}{c}{ MIC $(\boldsymbol{\mu g} \mathbf{g} \mathbf{m L})$} \\
\cline { 2 - 7 } & INH & RMP & EMB & STR & CPM & KAN & OFX & \\
\hline H37RV & S & S & S & S & S & S & S & 0.5 \\
FJ05349 & S & S & S & S & S & S & S & 0.5 \\
FJ05060 & S & S & S & S & S & S & S & 0.5 \\
FJ05195 & R & R & R & R & S & R & R & 4.0 \\
FJ05120 & R & R & S & S & S & S & S & 2.0 \\
FJ05189 & R & R & S & S & S & S & S & 1.0 \\
XZ & R & R & S & R & R & S & R & 4.0 \\
\hline
\end{tabular}

INH, isoniazid; RMP, rifampin; EMB, ethambutol; STR, streptomycin; CPM, cefpiramide; KAN, kanamycin; OFX, ofloxacin; MIC, minimum inhibitory concentrations; $S$, sensitive; $R$, resistant.

with the findings of DCS, the divergences of MIC values indicated that IMB-0283 inhibited Mtb bacteria in a DdlAdependent manner.

\section{Morphological Alterations of Mtb Treated With IMB-0283}

Morphological changes of Mtb treated with IMB-0283 were monitored to determine whether the inhibitor of DdlA altered the shape of Mtb. Mtb bacteria had a typical long-rod morphology resembling the log phase of bacteria at $12 \mathrm{~h}$ after treatment (Figures 6A,B). Shapes of these bacteria changed dramatically by 7 days after exposure to IMB-0283, during which they became shrunken, shorter, and fragmented (Figure 6C). These results indicate that IMB-0283 inhibited and killed Mtb by inhibiting cell-wall synthesis but not destroying mature cell walls.

\section{Cytotoxicity of IMB-0283}

Next, the $\mathrm{TC}_{50}$ values of IMB-0283 to HepG2 and Vero cells were evaluated (Table 3). Although the $\mathrm{TC}_{50}$ values of DCS for these mammalian cells were larger than $1,000 \mu \mathrm{g} / \mathrm{mL}$, the SI index of DCS was only about 80 . While the $\mathrm{TC}_{50}$ values of IMB-0283 for HepG2 and Vero cells were $115.4 \mu \mathrm{g} / \mathrm{mL}$ and $249.8 \mu \mathrm{g} / \mathrm{mL}$, respectively, the SI index of IMB-0283 was far greater than 200 . These results indicated that IMB-0283 might be safer than DCS.

\section{IMB-0283 Activity in a Mouse Model of TB}

Finally, the antibacterial activity of IMB-0283 in a mouse model of TB was evaluated (Figure 7). After 15 administrations (five times per week) at a dosage of $25 \mathrm{mg} / \mathrm{kg}$, INH reduced the Mtb load in mouse lungs from 5.58 to 3.29 (log CFU/mL), but DCS did not yield any antibacterial activity $(P>0.5)$ IMB-0283 significantly decreased the bacteria load to 4.40 , exhibiting much more potent antibacterial capacity than that of DCS $(P<0.0001)$.

\section{DISCUSSION}

The selection of a specific therapeutic target for high-throughput screening determines the output of lead compounds; hence,
A

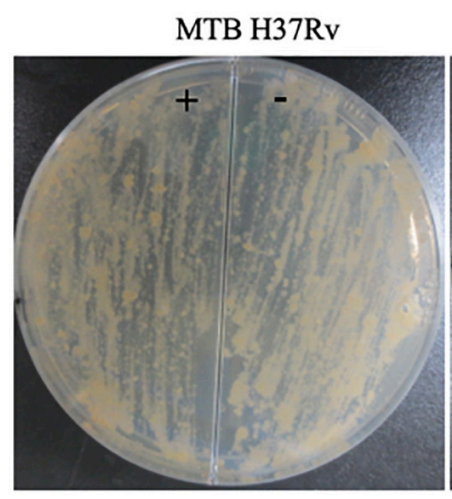

B

128
MTB-KD

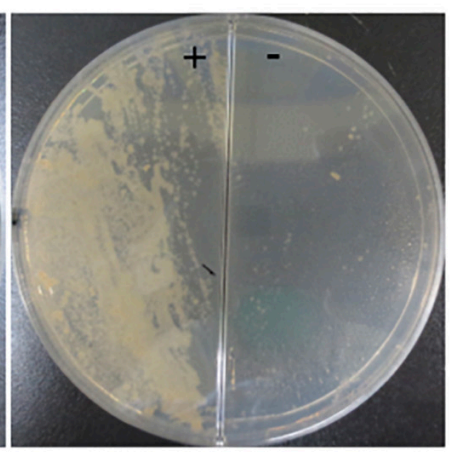

$0.0625(\mu \mathrm{g} / \mathrm{mL})$

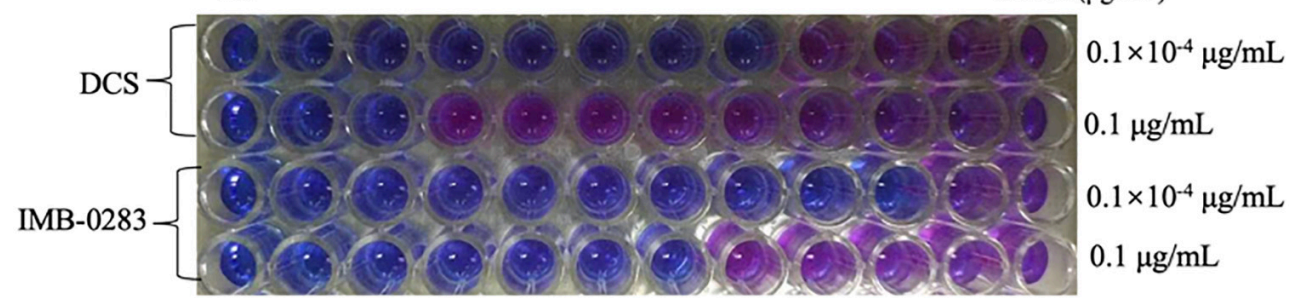

FIGURE 5 | DdlA is essential for Mtb growth and IMB-0283 inhibits Mtb growth in a DdlA-dependent manner. A conditional mutant strain, Mtb-KD, and its sensitivities to DCS and IMB-0283 were confirmed by culturing with different concentrations of pristinamycin. (A) Wild-type and mutant Mtb H37Rv were lineated on $7 \mathrm{H} 10$ solid medium in the presence $(+)$ or absence $(-)$ of $0.1 \mu \mathrm{g} / \mathrm{mL}$ of pristinamycin; the former formed lawns on the medium independent of the presence of the inducer, while the latter formed colonies only on the medium containing inducer. (B) DCS inhibited mutant Mtb at $1 \mu \mathrm{g} / \mathrm{mL}$ or $32 \mu \mathrm{g} / \mathrm{mL}$ when cultured with $0.1 \times 10^{-4} \mu \mathrm{g} / \mathrm{mL}$ (repressed) or $0.1 \mu \mathrm{g} / \mathrm{mL}$ (induced) of inducer. At the same conditions, IMB-0283 inhibited the mutant strain at either $0.25 \mathrm{or} 2 \mu \mathrm{g} / \mathrm{mL}$. 


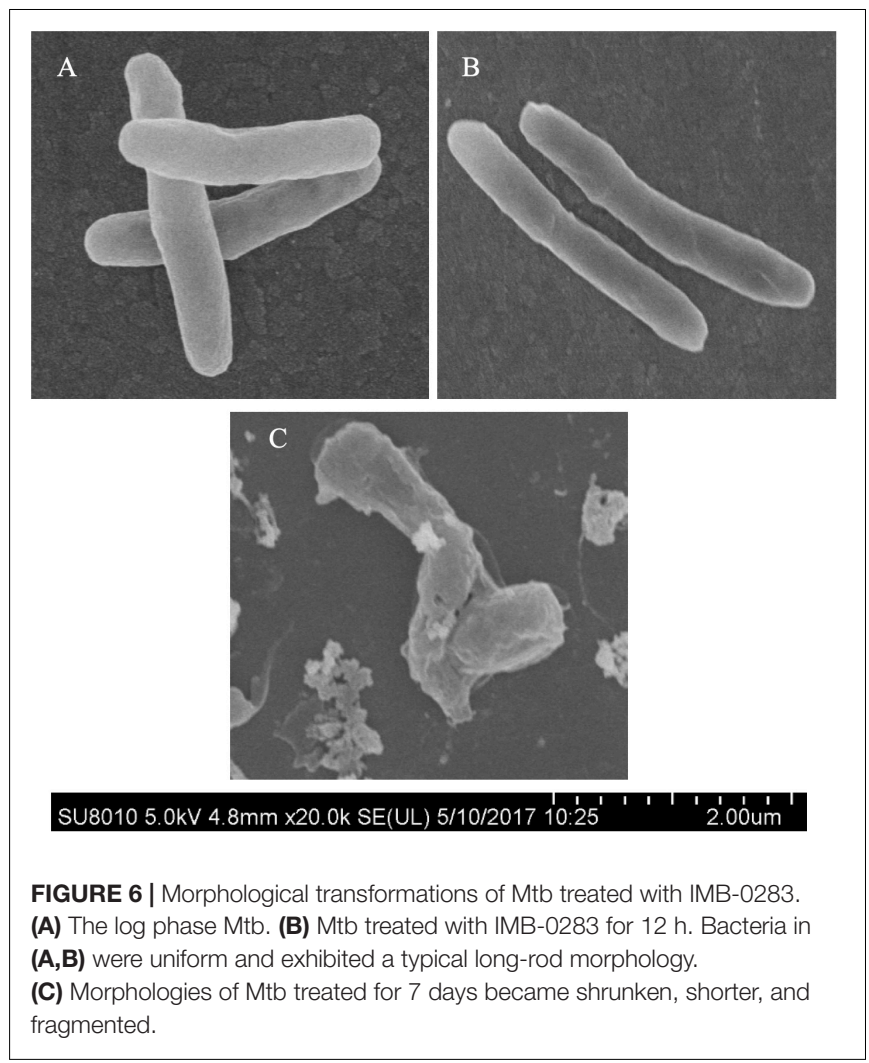

TABLE 3 | Cytotoxicity profiles of IMB-0283 compared with those of DCS.

\begin{tabular}{lcccccc}
\hline Compound & MTBH37Rv & \multicolumn{2}{c}{ HepG2 } & & \multicolumn{3}{c}{ Vero } \\
\cline { 3 - 4 } & MIC $(\mu \mathbf{g} / \mathbf{m L})$ & $\mathbf{T C}_{50}(\mu \mathbf{g} / \mathbf{m L})$ & SI & & TC $_{50}(\mu \mathbf{g} / \mathbf{m L})$ & SI \\
\hline DCS & 16 & 1380 & 86.25 & 2044 & 127.75 \\
IMB-0283 & 0.5 & 115.4 & 230.8 & 249.8 & 499.6
\end{tabular}

DCS, D-cycloserine; MIC, minimum inhibitory concentrations; SI, selective index which was calculated via $\mathrm{TC}_{50} / \mathrm{MIC}$.

it is questionable to establish high-throughput screening models based on essential gene products to search for inhibitors, as there are often considerable discrepancies between the essentiality and drugability of the resultant proteins (Gashaw et al., 2011). Therefore, we aimed to identify and test novel anti-mycobacterial compounds based on previously confirmed drug targets. The integrity of the cell wall is vital in supporting cell growth and virulence, as well as in influencing antimicrobial resistance (Abrahams and Besra, 2018). The processes for synthesizing and assembling cell walls have been targeted by several chemotherapeutics (Bhat et al., 2017). The reticular peptidoglycan of Mtb not only withstands exoteric osmotic pressure, but also provides anchor sites for other cell-wall components (Kieser and Rubin, 2014). The generation of peptidoglycans starts from the synthesis of intracellular glycopeptide precursors that are matured by conjugating with d-alanyl-d-alanine. The synthesis of this dipeptide can be interrupted by DCS, and DdlA has been shown to be the primary target of DCS

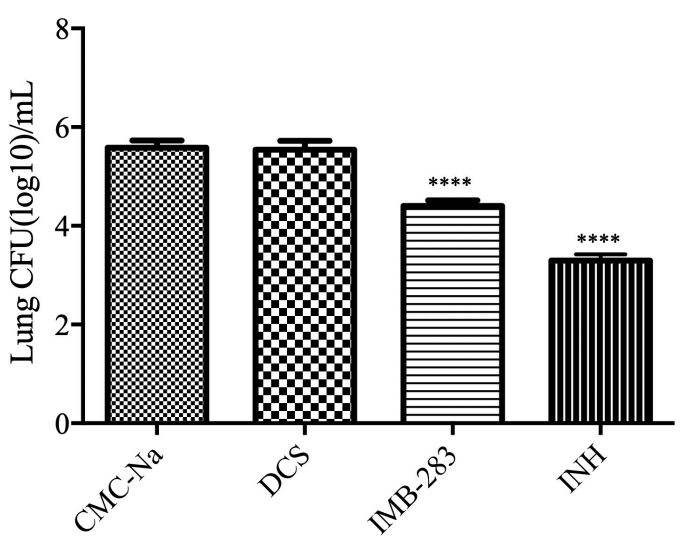

FIGURE 7 | Bacteriostatic activity of IMB-0283 in BALB/C mice. DCS, IMB-0283, and INH were each administered at $25 \mathrm{mg} / \mathrm{kg} 15$ times, and the vehicle CMC-Na was used as a negative control. INH and IMB-0283 reduces the bacterial load in lungs from 5.58 to 3.29 or 4.40 (log CFU/mL), respectively, but DCS showed no antibacterial activity $(P>0.5)$.

****Statistically significant difference from the negative control $(P<0.0001$; $n=6)$.

(Halouska et al., 2014). Although the severe side effects of DCS have limited its application in TB treatment, it has been demonstrated that DdlA is much more stable genetically than other anti-TB drug targets (McGrath et al., 2014). Therefore, researchers have attempted to exploit novel inhibitors that may supersede DCS (Wu et al., 2008; Triola et al., 2009; Vehar et al., 2011; Ameryckx et al., 2018). However, most screening assays have been carried out using DdlB of E. coli, the crystal structure of which is distinct from that of DdlA in Mtb (Bruning et al., 2011). Whether or not these inhibitors inhibit the growth of Mtb has not yet been reported. Consequently, there has been a continued need to search for novel inhibitors of Mtb DdlA specifically.

In the present study, a credible high-throughput screening model was established to search for specific inhibitors of Mtb DdlA from our chemical library. The cut-off value was set at $30 \%$ to obtain as many compounds as possible and to exclude ineffective inhibitors. Forty-eight compounds were obtained that had considerable inhibitory capacities to the Mtb enzyme. Among these chemicals, IMB-0283 competitively inhibited DdlA similar to that of DCS, and it nearly equally inhibited both standard and clinical drugresistant Mtb strains. IMB-0283 also exhibited bacteriostatic effects of both INH and RMP in vitro. We consulted PubChem for analogs of IMB-0283 for further information. There were 238 similar analogs that were obtained via PubChem, but little information regarding their biological activities were reported. This suggested that IMB-0283 may represent the first of this category to be found to inhibit Mtb. Future studies should test the inhibitory capacities of a series of several derivatives to DdlA of $\mathrm{Mtb}$ to analyze the structure-activity relationships of IMB-0283, which may provide clues to further optimize IMB-0283. Through 
measuring sensitivities of our conditional mutant strain, Mtb$\mathrm{KD}$, cultured with low or high inducers to IMB-0283, we found that IMB-0283 inhibited the catalytic activity of DdlA intracellularly. Although the larger divergences in enzymeinhibitory and antibacterial activities suggested that DdlA may not be the primary target of IMB-0283, IMB-0283 can interfere with the formation of cell walls but do not damage mature cell walls. Compared with properties of DCS, IMB-0283 exhibited less cellular toxicity and more bacteriostatic activity in a mouse model of TB.

Although the present study elucidated that IMB-0283 may represent a promising drug for the TB treatment, further research is needed to better understand its underlying mechanisms and to further confirm its low toxicity. In future studies, we plan to investigate the interactions of IMB-0283 with DdlA through crystallization, by which we may be able to optimize IMB-0283 in order to develop more potent and specific inhibitors. Additionally, we plan to screen for resistant Mtb strains to evaluate the probability to generate IMB-0283resistant mutations, as well as to expound upon mechanisms by which IMB-0283 inhibit Mtb. Lastly, comprehensive studies should be conducted to determine the adsorption, distribution, metabolism, excretion, and toxicity (ADMET) properties of these IMB-0283-modified compounds. Through these future studies, we expected to identify other promising anti-TB agents based on IMB- 0283 .

\section{DATA AVAILABILITY STATEMENT}

The raw data supporting the conclusions of this article will be made available by the authors, without undue reservation, to any qualified researcher.

\section{REFERENCES}

Abrahams, K. A., and Besra, G. S. (2018). Mycobacterial cell wall biosynthesis: a multifaceted antibiotic target. Parasitology 145, 116-133. doi: 10.1017/ s0031182016002377

Allen, B. W. (1998). "Mycobacteria: general culture methodology and safety considerations," in Mycobacteria Protocols, eds T. Parish, and N. G. Stoker (Totowa, NJ: Humana Press), 15-30. doi: 10.1385/0-89603-471-2:15

Ameryckx, A., Thabault, L., Pochet, L., Leimanis, S., Poupaert, J. H., Wouters, J., et al. (2018). 1-(2-Hydroxybenzoyl)-thiosemicarbazides are promising antimicrobial agents targeting D-alanine-D-alanine ligase in bacterio. Eur. J. Med. Chem. 159, 324-338. doi: 10.1016/j.ejmech.2018.09.067

Bhat, Z. S., Rather, M. A., Maqbool, M., Lah, H. U. L., Yousuf, S. K., and Ahmad, Z. (2017). Cell wall: a versatile fountain of drug targets in Mycobacterium tuberculosis. Biomed. Pharmacother. 95, 1520-1534. doi: 10.1016/j.biopha.2017. 09.036

Bruning, J. B., Murillo, A. C., Chacon, O., Barletta, R. G., and Sacchettini, J. C. (2011). Structure of the Mycobacterium tuberculosis D-alanine:Dalanine ligase, a target of the antituberculosis drug D-cycloserine. Antimicrob. Agents Chemother. 55, 291-301. doi: 10.1128/AAC.00 558-10

Correia, I., Adão, P., Roy, S., Wahba, M., Matos, C., Maurya, M. R., et al. (2014). Hydroxyquinoline derived vanadium(IV and V) and copper(II) complexes as potential anti-tuberculosis and anti-tumor agents. J. Inorg. Biochem. 141, 83-93. doi: 10.1016/j.jinorgbio.2014.07.019

\section{ETHICS STATEMENT}

The animal study was reviewed and approved by the Institutional Animal Care and Use Committee of the Institute of Medicinal Biotechnology.

\section{AUTHOR CONTRIBUTIONS}

JM and PG: experimental operation and manuscript preparation. XW and YG: data collection. YL: data analysis. CX: literature search and experimental design.

\section{FUNDING}

This study was supported by the National Major Scientific and Technological Special Project for "Significant New Drugs Development" (Grant No. 2015ZX09102007-009) and AMS Initiative for Innovative Medicine (Grant No. 2016-I2M-1-013), the National Natural Science Foundation of Chinese (81803412 and 81903678), and the Fundamental Research Funds for Central Public-interest Scientific Institution (Centre for Tuberculosis) (Grant No. 2017PT31010).

\section{ACKNOWLEDGMENTS}

We are very grateful to Prof. Chuanyou Li (Beijing Chest Hospital, Capital Medical University and Beijing Tuberculosis and Thoracic Tumor Research Institute, China) for his guidance and assistance in the manipulation of Mycobacterium tuberculosis.

Darby, C. M., and Nathan, C. F. (2010). Killing of non-replicating Mycobacterium tuberculosis by 8-hydroxyquinoline. J. Antimicrob. Chemother. 65, 1424-1427. doi: $10.1093 / \mathrm{jac} / \mathrm{dkq} 145$

Forti, F., Crosta, A., and Ghisotti, D. (2009). Pristinamycin-inducible gene regulation in mycobacteria. J. Biotechnol. 140, 270-277. doi: 10.1016/j.jbiotec. 2009.02.001

Gashaw, I., Ellinghaus, P., Sommer, A., and Asadullah, K. (2011). What makes a good drug target? Drug Discov. Today 16, 1037-1043. doi: 10.1016/j.drudis. 2011.09.007

Gordhan, B. G., and Parish, T. (2001). "Gene replacement using pretreated DNA," in Mycobacterium Tuberculosis Protocols, eds T. Parish, and N. G. Stoker, (Totowa, NJ: Humana Press), 77-92. doi: 10.1385/1-59259147-7:077

Halouska, S., Fenton, R. J., Zinniel, D. K., Marshall, D. D., Barletta, R. G., and Powers, R. (2014). Metabolomics analysis identifies D-alanine-D-alanine ligase as the primary lethal target of D-cycloserine in Mycobacteria. J. Proteome Res. 13, 1065-1076. doi: 10.1021/pr4010579

Iversen, P. W., Eastwood, B. J., Sittampalam, G. S., and Cox, K. L. (2006). A comparison of assay performance measures in screening assays: signal window, $\mathrm{Z}^{\prime}$ factor, and assay variability ratio. J. Biomol. Screen. 11, 247-252. doi: 10.1177/ 1087057105285610

Kass, J. S., and Shandera, W. X. (2010). Nervous system effects of antituberculosis therapy. CNS Drugs 24, 655-667. doi: 10.2165/11534340-000000000-00000

Kieser, K. J., and Rubin, E. J. (2014). How sisters grow apart: mycobacterial growth and division. Nat. Rev. Microbiol. 12, 550-562. doi: 10.1038/nrmicro3299 
McGrath, M., Gey van Pittius, N. C., van Helden, P. D., Warren, R. M., and Warner, D. F. (2014). Mutation rate and the emergence of drug resistance in Mycobacterium tuberculosis. J. Antimicrob. Chemother. 69, 292-302. doi: 10.1093/jac/dkt364

Meng, J. Z., Yang, Y. H., Xiao, C. L., Guan, Y., Hao, X. Q., Deng, Q., et al. (2015). Identification and validation of aspartic acid semialdehyde dehydrogenase as a new anti-mycobacterium tuberculosis target. Int. J. Mol. Sci. 16, 23572-23586. doi: 10.3390/ijms161023572

Montoro, E., Lemus, D., Echemendia, M., Martin, A., Portaels, F., and Palomino, J. C. (2005). Comparative evaluation of the nitrate reduction assay, the MTT test, and the resazurin microtitre assay for drug susceptibility testing of clinical isolates of Mycobacterium tuberculosis. J. Antimicrob. Chemother. 55, 500-505. doi: $10.1093 /$ jac/dki023

Prosser, G. A., and de Carvalho, L. P. S. (2013). Kinetic mechanism and inhibition of Mycobacterium tuberculosis $\mathrm{D}$-alanine:D-alanine ligase by the antibiotic D-cycloserine. FEBS J. 280, 1150-1166. doi: 10.1111/febs.12108

Shen, G. H., Wu, B. D., Hu, S. T., Lin, C. F., Wu, K. M., and Chen, J. H. (2010). High efficacy of clofazimine and its synergistic effect with amikacin against rapidly growing mycobacteria. Int. J. Antimicrob. Agents 35, 400-404. doi: 10.1016/j.ijantimicag.2009.12.008

Triola, G., Wetzel, S., Ellinger, B., Koch, M. A., Hübel, K., Rauh, D., et al. (2009). ATP competitive inhibitors of D-alanine-D-alanine ligase based on protein kinase inhibitor scaffolds. Bioorg. Med. Chem. 17, 1079-1087. doi: 10.1016/j bmc.2008.02.046

Vehar, B., Hrast, M., Kovaè, A., Konc, J., Mariner, K., Chopra, I., et al. (2011). Ellipticines and 9-acridinylamines as inhibitors of D-alanine:D-alanine ligase. Bioorg. Med. Chem. 19, 5137-5146. doi: 10.1016/j.bmc.2011.07.020

WHO, (2018). Global Tuberculosis Report 2018. Geneva: World Health Organization.

Wu, D. L., Kong, Y. H., Han, C., Chen, J., Hu, L. H., Jiang, H. L., et al. (2008). $\mathrm{D}$-alanine:D-alanine ligase as a new target for the flavonoids quercetin and apigenin. Int. J. Antimicrob. Agents 32, 421-426. doi: 10.1016/j.ijantimicag.2008. 06.010

Conflict of Interest: The authors declare that the research was conducted in the absence of any commercial or financial relationships that could be construed as a potential conflict of interest.

Copyright (c) 2020 Meng, Gao, Wang, Guan, Liu and Xiao. This is an open-access article distributed under the terms of the Creative Commons Attribution License (CC BY). The use, distribution or reproduction in other forums is permitted, provided the original author(s) and the copyright owner(s) are credited and that the original publication in this journal is cited, in accordance with accepted academic practice. No use, distribution or reproduction is permitted which does not comply with these terms. 